\title{
Can Smaller-Scale Comprehensive Cancer Centers Provide Outstanding Care in Abdominal and Thoracic Pediatric Solid Tumor Surgery? Results of a 14-Year Retrospective Single-Center Analysis
}

\author{
J. M. Joseph, MD', A. M. Farron, $\mathrm{MD}^{1}$, R. Renella, $\mathrm{MD}, \mathrm{PhD}^{1,2}$, and C. Gapany, $\mathrm{MD}^{3}$ \\ ${ }^{1}$ Division of Pediatric Surgery, Department of Pediatrics and Pediatric Surgery (DMCP), University Hospital of Lausanne, \\ Lausanne, Switzerland; ${ }^{2}$ Boston Children's Hospital-Dana-Farber Cancer Institute, Harvard Medical School, Boston, \\ MA; ${ }^{3}$ Department of Pediatrics and Pediatric Surgery, University Hospital of Geneva, Geneva, Switzerland
}

\begin{abstract}
Purpose. Quality of care and its measurement represent a considerable challenge for pediatric smaller-scale comprehensive cancer centers (pSSCC) providing surgical oncology services. It remains unclear whether center size and/or yearly case-flow numbers influence the quality of care, and therefore impact outcomes for this population of patients.

Patients and Methods. We performed a 14-year, retrospective, single-center analysis, assessing adherence to treatment protocols and surgical adverse events as quality indicators in abdominal and thoracic pediatric solid tumor surgery.

Results. Forty-eight patients, enrolled in a research-associated treatment protocol, underwent 51 cancer-oriented surgical procedures. All the protocols contain precise technical criteria, indications, and instructions for tumor surgery. Overall, compliance with such items was very high, with 997/1,035 items (95\%) meeting protocol requirements. There was no surgical mortality. Twenty-one patients $(43 \%)$ had one or more complications, for a total of 34 complications (66\% of procedures). Overall, $85 \%$ of complications were grade 1 or 2 according to ClavienDindo classification requiring observation or minor
\end{abstract}

R. Renella and C. Gapany share the senior authorship.

\section{(C) Society of Surgical Oncology 2014}

First Received: 2 December 2013; Published Online: 10 January 2014

J. M. Joseph, MD

e-mail: jean-marc.joseph@chuv.ch medical treatment. Case-sample and outcome/effectiveness data were comparable to published series. Overall, our data suggest that even with the modest caseload of a pSSCC within a Swiss tertiary academic hospital, compliance with international standards can be very high, and the incidence of adverse events can be kept minimal.

Conclusion. Open and objective data sharing, and discussion between pSSCCs, will ultimately benefit our patient populations. Our study is an initial step towards the enhancement of critical self-review and quality-of-care measurements in this setting.

The objective measurement of quality of care is crucial for the improvement of services provided to children with cancer. In Switzerland, as in other European countries, there is a reflection on centralization of highly specific healthcare services, such as pediatric oncology. In this setting, we found it essential to monitor the quality of the services provided at a given time.

Unfortunately, obtaining reliable data on performance, safety, and overall outcomes represents a major challenge in pediatric surgical oncology, even more so in low-output, but nonetheless reference/tertiary, hospitals. This issue particularly affects pediatric smaller-scale comprehensive cancer centers (pSSCC). Globally, it remains unclear which specific determinants, either in size or related overall case turnover of the providing center, directly or indirectly influence outcomes. Moreover, comparisons between pediatric surgical oncology centers remain particularly difficult due to (a) the rarity of the diseases managed, all of which are classifiable as orphan; ${ }^{1,2}$ and (b) the extreme diversity in the encountered clinical spectrum. 
The dilemma is whether minimal requirements in case numbers and/or mere center size criteria (i.e. in number of certified dedicated physicians or nursing staff) should be added to published guidelines. ${ }^{3}$ In fact, it is undoubtedly essential not only for the patients, but also for the rational use of medical and financial resources, that pSSCC meet proper standards of care, and achieve results comparable to larger centers.

As the economical impact of healthcare has become a more vigorous modulator of political decisions, it is imperative that smaller institutions address the question of quality in pediatric surgical oncology, and provide evidence to support or modify the existing lines of practice. In fact, while larger institutions have the privilege of experience from the abundance of treated cases, it remains to be proven that outstanding care can be provided at a smaller scale.

Solid malignant tumors account for 35-40\% of all pediatric malignancies, and make up to $50 \%$ of cancerrelated deaths. ${ }^{4,5}$ Neuroblastoma, renal tumor, and other abdominal or thoracic tumors account for 6, 5.2 \%, and less than $1 \%$, respectively, of pediatric cancers according to the Swiss Childhood Cancer Registry. ${ }^{6}$ International research-associated treatment protocols have helped reduce these dim figures, as exemplified by improved survival and lower morbidity in neuroblastoma after implementation of the results of multicentric studies. ${ }^{7,8}$ Complementary to other treatment modalities, surgical excision is often an important step in the management of these tumors.

The extent to which eligible children are actually included in research-associated treatment protocols is usually not reported in the literature, and should be regarded as an indicator of quality of care in pediatric oncology patients, similar to compliance with published guidelines in other fields of healthcare. ${ }^{9}$ Adverse event rates are another important quality indicator. Cancer is a systemic disease and pediatric surgical oncology is often performed on children in physiological distress, therefore more prone to complications. Yet again, little has been published on this issue, which may be associated with negative values in medical and surgical tradition, instead of being an incentive for improvement. Notwithstanding the existing controversy regarding the use and definitions of 'complications', 'adverse events', or 'adverse occurrences', these phrasings will be used interchangeably in this work. ${ }^{10}$

In Switzerland, all pediatric oncology patients are referred, without pre-selection, to one of the five pediatric cancer centers such as ours which are equipped to manage all complex cases. With this single-center, retrospective study spanning 13 years of practice, we desired to critically address the compliance of our unit to enrollment into standard-of-care treatment protocols, and measure the rate of adverse events observed in our patient population over the last 13 years. The hypothesis was that these two indicators could allow basic but reliable comparisons with larger centers and serve as proxies for quality of care. Survival data were also assessed for comparison purposes.

\section{PATIENTS AND METHODS}

The study protocol was approved by the Lausanne University Research Ethics Committee for Human Research (number 217/11, 10 June 2011). Written consent was given by the patients for their information to be stored in the hospital database and used for research.

Medical and surgical files of all children $<18$ years of age who were operated on in our institution between 1 January 1997 and 31 December 2010 were searched both electronically and manually for diagnosis of neuroblastoma, nephroblastoma, primary neuroectodermal tumor (PNET) or other abdominal and thoracic primary solid malignancies, as well as for surgical codes. Patients with a differential diagnosis of malignant tumor who ended up having a benign mass were included up to the point of histological diagnosis of a non-malignant process. Simple biopsies were not included. Patients with central nervous system (CNS) tumors were excluded, as were patients operated primarily for excision or staging in another institution and referred secondarily. For more homogeneity, patients with germinal tumors were not included. All patients were managed and/or supervised by one dedicated pediatric oncology surgeon.

Patients' records were then checked for inclusion in a protocol, and for accordance to every single identifiable surgical item of each effective protocol. The protocol details are listed in Table 2.

Compliance with non-surgical directives was not recorded or analyzed. Overall compliance was summarized as the proportion of items satisfactorily verified to the total number of items. Rates are expressed in percentages, and ages are expressed as medians.

Early (i.e. within 30 days of surgery) and late complications, defined as deviations from the ideal postoperative course, were identified with the help of diagnostic codes and through manual searching, and graded according to Clavien and Dindo. ${ }^{11}$

Primary endpoints were (1) inclusion into an international protocol; (2) adherence to the effective protocol; (3) surgical mortality; and (4) complication/adverse event rates. Secondary endpoints were (1) completeness of surgical tumor removal when indicated; (2) time to enteral feeding; (3) possible delay of postoperative chemotherapy when required; and (4) survival. 
TABLE 1 Clinical and demographic data of the study population

\begin{tabular}{|c|c|c|c|c|}
\hline & $\begin{array}{l}\text { Total } \\
{[n(\%)]}\end{array}$ & $\begin{array}{l}\text { Included } \\
{[n(\%)]}\end{array}$ & $\begin{array}{l}\text { Survival } \\
{[n(\%)]}\end{array}$ & $\begin{array}{l}\text { Event- } \\
\text { free } \\
\text { survival } \\
{[n(\%)]}\end{array}$ \\
\hline No. of patients & $61(100)$ & $50(100)$ & $42(84)$ & $39(78)$ \\
\hline M:F ratio & 0.8 & 0.9 & - & - \\
\hline $\begin{array}{l}\text { Median age at } \\
\text { presentation }\end{array}$ & $\begin{array}{l}1 \text { year } \\
10 \text { months }\end{array}$ & $\begin{array}{l}2 \text { years } \\
3 \text { months }\end{array}$ & - & - \\
\hline Neuroblastoma & $30(50)$ & $22(44)$ & $16(72)$ & $14(63)$ \\
\hline $\begin{array}{l}\text { Nephroblastoma } \\
\text { (Wilms tumor) }\end{array}$ & $12(18)$ & $12(24)$ & 12 & $11(91)$ \\
\hline Ganglioneuroma & $6(10)$ & $4(8)$ & 4 & 4 \\
\hline PNET/Ewing & $5(9)$ & $4(8)$ & 4 & 4 \\
\hline Ganglioneuroblastoma & $2(3)$ & $2(4)$ & 2 & 2 \\
\hline Rhabdomyosarcoma & $2(3)$ & $2(4)$ & 1 & 1 \\
\hline $\begin{array}{l}\text { Renal clear cell } \\
\text { carcinoma }\end{array}$ & $1(2)$ & $1(2)$ & 1 & 1 \\
\hline Renal fibroadenoma & $1(2)$ & $1(2)$ & 1 & 1 \\
\hline $\begin{array}{l}\text { Adrenocortical } \\
\text { carcinoma }\end{array}$ & $1(2)$ & $1(2)$ & 1 & 1 \\
\hline Renal sarcoma & $1(2)$ & $1(2)$ & 0 & 0 \\
\hline
\end{tabular}

$M$ male, $F$ female, $P N E T$ primary neuroectodermic tumor

\section{RESULTS}

Sixty-one eligible patients were identified, of whom 50 underwent 53 cancer-oriented surgical procedures and were included in the study population. Clinical and demographic data are described in Table 1.

All operations were performed by, or under direct supervision of, a senior pediatric oncology surgeon. All operating decisions were discussed in a tumor board meeting including a pediatric oncologist, a dedicated pediatric radiologist, and a pediatric oncology surgeon.

Forty-eight patients included in our analysis were enrolled in one of the 16 research-associated treatment protocols (Table 2), which contain precise technical criteria, indications, and instructions for tumor surgery. The remaining two patients had renal sarcoma and adrenocortical carcinoma, for which there are no such protocols. These two patients were analyzed for postoperative complications only.

Overall, compliance with such items was very high, with 997/1,053 items (95\%) meeting protocol requirements. When analyzed by protocol, all patients enrolled in eight of the 16 treatment protocols $(50 \%)$ had full (i.e. $100 \%$ ) compliance with surgical requirements, and constituted $63 \%$ of the overall procedures $(n=32 / 51)$.

Problematic items concerned mainly the documentation of the required lymphatic node sampling in the operative reports, more particularly the documentation of staging
TABLE 2 Compliance with surgical protocols

\begin{tabular}{|c|c|c|c|}
\hline Protocols & $\begin{array}{l}\text { No. of } \\
\text { patients } \\
\text { enrolled }\end{array}$ & $\begin{array}{l}\text { No. of } \\
\text { procedures }\end{array}$ & $\begin{array}{l}\text { Overall compliance to } \\
\text { surgical items in the } \\
\text { protocol (\% of items } \\
\text { satisfactorily addressed) }\end{array}$ \\
\hline $\begin{array}{l}\text { Neuroblastoma } \\
\text { group }\end{array}$ & 28 & 30 & \\
\hline Protocol HR/ESIOP & 4 & 6 & 90 \\
\hline Protocol LNESG2 & 8 & 8 & 92 \\
\hline Protocol INES & 5 & 5 & 93 \\
\hline Protocol SIOP E.N. & 2 & 2 & 95 \\
\hline Protocol 3961 & 2 & 2 & 93 \\
\hline Protocol POG 9640 & 2 & 2 & 100 \\
\hline Protocol 9641 & 1 & 1 & 85 \\
\hline Protocol NB87 & 2 & 2 & 100 \\
\hline Protocol NB90 & 2 & 2 & 100 \\
\hline $\begin{array}{l}\text { Nephroblastoma } \\
\text { group }\end{array}$ & 14 & 15 & \\
\hline Protocol POG 9440 & 4 & 4 & 90 \\
\hline Protocol SIOP 2001 & 10 & 11 & 99 \\
\hline PNET & 4 & 4 & \\
\hline $\begin{array}{l}\text { Protocol Euro- } \\
\text { Ewing } 99\end{array}$ & 2 & 2 & 100 \\
\hline $\begin{array}{l}\text { Protocol } \\
\text { AEWSOO31 }\end{array}$ & 1 & 1 & 100 \\
\hline Protocol POG 9457 & 1 & 1 & 100 \\
\hline Rhabdomyosarcoma & 2 & 2 & \\
\hline Protocol RMS 2005 & 1 & 1 & 100 \\
\hline $\begin{array}{l}\text { Protocol } \\
\quad \text { ARSTO331 }\end{array}$ & 1 & 1 & 100 \\
\hline Overall & 48 & 51 & 96 \\
\hline
\end{tabular}

PNET primary neuroectodermic tumor

(18/38 problematic items; $47 \%)$. Such observation was made when pathology reports, documenting lymph node samples that were analyzed, were compared with the surgeon's operative notes. This discrepancy did not affect treatment decisions for any of the patients in our series.

There was no surgical mortality and no intensive care unit admission, except for planned postoperative follow-up. Twenty-one patients $(43 \%)$ had one or more complications, for a total of 34 complications (66\% of procedures). Overall, $85 \%$ of complications were grade 1 or 2 according to Clavien and Dindo. ${ }^{11}$ The most frequent complication was postoperative fever, with ten occurrences. Documented infections occurred in two cases: one central line infection with Staphylococcus epidermidis and one urinary tract infection with Escherichia coli. In one further case of clinical infection, S. epidermidis was found on the gastrostomy but was not considered relevant. There were no cases of sepsis. The other complications were single occurrences. Out of eight patients who received a 
TABLE 3 Details, grades, and rates of complications according to Clavien and Dindo

\begin{tabular}{lll}
\hline & $N$ & Rate $(\%)$ \\
\hline No. of patients & 48 & \\
No. of procedures & 51 & \\
No. of complications & 34 & 66 (of patients) \\
Grade 1 & 19 & 56 (of complications) \\
Grade 2 & 10 & 29 \\
Grade 3a & 0 & 0 \\
Grade 3b & 3 & 9 \\
Grade 4a & 2 & 6 \\
Grade 4b & 0 & 0 \\
Grade 5 & 0 & 0 \\
\hline
\end{tabular}

TABLE 4 Details of complications

\begin{tabular}{lcc}
\hline Type of complication & No. of cases & $\begin{array}{l}\text { Percentage of } \\
\text { cases }\end{array}$ \\
\hline Fever $\left(\geq 38.5^{\circ} \mathrm{C}\right)$ & 10 & 21 \\
Documented infection & 4 & 8 \\
Intestinal necrosis & 1 & 2 \\
Infection/wound breakdown & 1 & 2 \\
Subileus & 5 & 10 \\
Need for oxygen supplementation & 1 & 2 \\
Abdominal compartment & 1 & 2 \\
$\quad$ syndrome & & \\
Cardiac arrhythmia & 1 & 2 \\
Pleural effusion & 1 & 2 \\
Pneumothorax & 1 & 2 \\
Subcutaneous emphysema & 1 & 2 \\
Chronic renal failure grade 1 & 1 & 2 \\
Acute tubular necrosis & 1 & 2 \\
Hematuria & 1 & 2 \\
Lower limb edema & 1 & 2 \\
Ulnar nerve compression & 1 & 2 \\
Phrenic nerve injury & 1 & 2 \\
Hyponatremia & 1 & 2 \\
\hline & &
\end{tabular}

blood transfusion in the perioperative period, only one did so because of massive surgical hemorrhage. All the other blood transfusions were motivated by preoperative chemotherapy-induced anemia.

Macroscopic gross total surgical excision was complete in $40 / 48$ patients $(83 \%)$. Incomplete macroscopic resection $(n=7)$ was found in stage III or IV neuroblastoma $(n=5)$ and ganglioneuroma encasing the aorta or iliac vessels $(n=2)$.

One patient experienced 180 days of pseudo-intestinal obstruction after intestinal ischemia during Wilms tumor resection and concomitant vincristine chemotherapy. Postoperative pleural effusion required chest tube insertion in two cases. Table 3 summarizes the complications encountered and their grades according to Clavien and Dindo. ${ }^{11}$ Table 4 details the complications.

Median time to enteral feeding was 1 day (mean 11 days). Median time to postoperative chemotherapy was 15 days (mean 16.2).

Outcomes for each type of tumor are summarized in Table 1 and are detailed as follows:

There were 30 identified cases of neuroblastoma; twenty-two required primary tumor resection and were included in the study. Surgery was the sole treatment for five patients with localized disease; all survived. The other 17 patients were included in infant or high-risk protocols. Fifteen of these patients had stage III or IV requiring neoadjuvant chemotherapy. Nine survived, giving a survival for stage III and IV tumors estimated at approximately $60 \%$, and $53 \%$ for stage IV alone. Four died within 2 years of diagnosis, one within 5 years and one after 5 years, all with stage IV disease without local recurrence.

Among non-included patients, five survived without surgery, two died of tumor progression under chemotherapy, and one patient died of late metastatic disease. These three patients were not candidates for surgery.

All patients with ganglioneuroma, ganglioneuroblastoma, PNET, Wilms tumor, renal clear cell carcinoma, renal fibroadenoma, or adrenocortical carcinoma survived. One patient with Wilms tumor needed a second operation for a local recurrence. The patient with renal sarcoma died 6 years after diagnosis of metastatic disease without local recurrence.

One patient with pelvic rhabdomyosarcoma needed brachytherapy for local control and is still alive more than 3 years after diagnosis. The other patient with RMS of the abdominal wall died of metastatic disease within 6 months of diagnosis.

\section{DISCUSSION}

The aim of the study was to analyze retrospectively the enrollment into standard-of-care treatment protocols, and measure the rate of adverse events observed in our patient population. We were able to observe that over 13 years of pediatric solid tumor surgeries in a pSSCC in Switzerland, all patients were included into international treatment protocols. Compliance with the itemized sections of surgical requirements in these protocols was very high (96\%), and the rate of gross-total resections was $85 \%$. Complete macroscopic excision was not always the ultimate goal of surgery. For e.g. when efforts for complete removal could 
jeopardize the patient's safety with no known benefit in survival. We noted the absence of any fatal outcome directly linked to a surgical cancer-directed procedure. Surgery did not delay further treatment as our patients were ready for chemotherapy, when required, within an average of 15 days from surgery. Overall, our data seems to suggest that even with the modest caseload of a pSSCC within a Swiss tertiary academic hospital, compliance with international standards can reach a high level. This is an important finding, especially as it is known how difficult it can be to implement recommendations consistently. ${ }^{12}$

Interestingly, while risk-stratification was not the purpose of our study, we found our case-mix not to favor lower complexity cases. In fact, as neural crest-derived tumors are the most frequent thoracic and abdominal tumors in children, they represented the larger fraction of cases in this study $(n=28)$. Only eight cases $(28 \%)$ were treated according to the LNSG2 protocol (localized L1, using the new image-defined risk factor classification), as indicated in Table 2; the other 20 cases (72\%) were more complex cases. In comparison, in a recent Children's Oncology Group review, ${ }^{13}$ the low-risk group comprised $50 \%$ of cases.

Survival for local neuroblastoma can be estimated at $100 \%$. Survival for stage III and IV was 60 and $53 \%$ for metastatic cases. Surgery was complete for $8 / 15$ patients with stage III and IV neuroblastomas. Incomplete resection was made in accordance with the protocol in the remaining $7 / 15$ patients. Only one of these seven patients required two further surgical interventions for recurrences at the limit of the irradiation field. Irradiation of the primary tumor site is mandatory in all high-risk neuroblastoma protocols.

All but one of the patients with Wilms tumor survived event-free after 4 years of follow-up, regardless of the stage of the tumor (stage I, $n=4$; stage II, $n$ 4; stage III, $n=2$; stage IV, $n=1$; and stage $\mathrm{V}, n=1$ )

Despite the small figures that hamper any tentative generalization, we feel that our case-mix and survival data compare fairly to data recently published by larger centers. $^{14,15}$

We believe our findings reflect the existence of a compulsory and regular multidisciplinary tumor-board discussion in our center, leading to consensual and peerand openly-discussed treatment strategies, as well as continuous postgraduate surgical education. In fact, it has been recognized in the literature that such procedural steps are very important, as defective planning can lead to increased morbidity and mortality in cancer patients. ${ }^{16}$

Our analysis has several limitations. Its retrospective nature and the limited number of cases render any analysis difficult to interpret. Regarding our adverse event analysis, we deliberately chose to use a stringent definition of surgical complications, as defined by Clavien and Dindo, ${ }^{11}$ which has the advantage of including all deviations from the ideal postoperative course. As expected, using this classification, our complication rate was accordingly high with 34 patients out of $48(66 \%)$. Fortunately, severe complications were rare, with $85 \%$ being Clavien grade 1 or 2 , requiring no or simple medical treatment. Surgical management of complications was needed for chest tube insertion in two cases. The single most frequent complication was postoperative fever without clinical infection, which is not surprising in oncology patients. The rate of ten occurrences in 51 procedures $(19.6 \%)$ found in our series is actually very low compared with reported figures of up to $79 \%$ in another retrospective pediatric study. ${ }^{17}$

Our data also highlight several important improvement opportunities. Pre-existing frailness is but one explanation for the adverse events observed, but certainly does not account for the occurrence of pneumothorax or ulnar nerve compression. Although of no consequence to the patients, lymphatic node sampling documentation in the operative report was clearly below our expectations. This will require an adaptation of our practice, through the compulsory use of a protocol-specific intra-operative checklist and diseasespecific standardized surgical reporting forms clearly prompting description of the nodal dissection status. The impact for the patients of this specific weak point in our documentation was limited, as the staging could be adequately retrieved from the histological examination reports in retrospect. This data reveals that surgery was properly performed even if the surgical report was imperfect. In 2005, the National Wilms Tumor Study Group reported $9 \%$ of the required lymph node sampling not being performed, ${ }^{18}$ which may have a significant impact on tumor staging and hence therapy.

Conceptually, there are several advantages of care within a pSSCC, to which our positive data could hint. At baseline, a recent study in the field of pediatric brain tumors showed no evidence that the quality of care differed between smaller and larger centers. ${ }^{19}$ In addition, one might argue that the size of our unit might facilitate the rapid, smooth, and effective implementation and updating of surgical recommendations, and therefore directly positively impact quality of care. In addition, the small human resource turnover, and close-up surgical education of younger colleagues, could contribute to overall improved services to our patient population. The disadvantages certainly include the limited numbers of cases, which negatively influences the time required to build a solid pool of experience for such complex and highly specialized procedures. In such settings, recruitment and training of younger surgeons and their long-term mentoring is paramount. All of the above considerations have a clearly speculative nature, but well-designed studies by smaller-scale pediatric surgical oncology units could be performed to answer them. 
The small throughput in institutions such as ours makes it hard to reach statistical significance in outcome studies outside international protocols, and this was not the aim of our work. However the adherence to these protocols can be quite easily monitored as one quality indicator, along with the rate of undesired events. We feel that our study could serve as an example to foster open and objective datasharing between pSSCCs, and enhance critical self-review and quality of care measurements to ultimately benefit our patient populations.

\section{CONCLUSION}

In our 14-year retrospective study assessing adherence to treatment protocols and surgical adverse events as quality indicators in abdominal and thoracic pediatric solid tumor surgery we identified a high rate of adhesion to surgical protocols, few serious complications, and no surgical mortality. Our data suggests that meticulous surgical planning and execution by dedicated oncologic pediatric surgeons, and tight collaboration with pediatric oncologists, are absolute prerequisites for quality in our care. Under these conditions, even pSSCC can meet the standards set by larger, leading centers.

ACKNOWLEDGEMENT The authors would like to thank Dr. Emma Garcia for her help in data collection.

DISCLOSURE The authors declare that they have no conflicts of interest in this study, or any financial and personal relationships with other people or organizations that could inappropriately influence the work.

\section{REFERENCES}

1. Fernandez CV. Our moral obligations in caring for patients with orphan cancers. CMAJ. 2007;176(3):297.

2. Vogelzang NJ, Benowitz SI, Adams S, Aghajanian C, Chang SM, Dreyer ZE, et al. Clinical cancer advances 2011: annual report on progress against cancer from the American Society of Clinical Oncology. J Clin Oncol. 2012;30(1):88-109.

3. Corrigan JJ, Feig SA; American Academy of Pediatrics. Guidelines for pediatric cancer centers. Pediatrics 2004;113(6):1833-5.

4. Kaatsch P. Epidemiology of childhood cancer. Cancer Treat Rev. 2010;36(4):277-85.

5. Somasundaram R, Herlyn D. Chemokines and the microenvironment in neuroectodermal tumor-host interaction. Semin Cancer Biol. 2009;19(2):92-6.
6. Michel G, von der Weid NX, Zwahlen M, Adam M, Rebholz CE, Kuehni CE, et al. The Swiss childhood cancer registry: rationale, organisation and results for the years 2001-2005. Swiss Med Wkly. 2007;137(35-36):502-9.

7. Matthay KK, Villablanca JG, Seeger RC, Stram DO, Harris RE, Ramsay NK, et al. Treatment of high-risk neuroblastoma with intensive chemotherapy, radiotherapy, autologous bone marrow transplantation, and 13-cis-retinoic acid. Children's Cancer Group. N Engl J Med. 1999;341(16):1165-73.

8. Yu AL, Gilman AL, Ozkaynak MF, London WB, Kreissman SG, Chen HX, et al. Anti-GD2 antibody with GM-CSF, interleukin-2, and isotretinoin for neuroblastoma. N Engl J Med. 2010;363(14): 1324-34.

9. Morse RB, Hall M, Fieldston ES, McGwire G, Anspacher M, Sills MR, et al. Hospital-level compliance with asthma care quality measures at children's hospitals and subsequent asthmarelated outcomes. JAMA. 2011;306(13):1454-60.

10. Dekutoski MB, Norvell DC, Dettori JR, Fehlings MG, Chapman JR. Surgeon perceptions and reported complications in spine surgery. Spine. 2010;35(9 Suppl):S9-S21.

11. Clavien PA, Barkun J, de Oliveira ML, Vauthey JN, Dindo D, Schulick RD, et al. The Clavien-Dindo classification of surgical complications: five-year experience. Ann Surg. 2009;250(2): 187-96.

12. Lee HY, Cooke CE, Robertson TA. Use of secondary prevention drug therapy in patients with acute coronary syndrome after hospital discharge. J Manag Care Pharm. 2008;14(3):271-80.

13. Strother DR, London WB, Schmidt ML, Brodeur GM, Shimada $\mathrm{H}$, Thorner $\mathrm{P}$, et al. Outcome after surgery alone or with restricted use of chemotherapy for patients with low-risk neuroblastoma: results of Children's Oncology Group study P9641. J Clin Oncol. 2012;30(15):1842-8.

14. Hall G, Grant R, Weitzman S, Maze R, Greenberg M, Gerstle JT. Predictors of surgical outcome in Wilms' tumor: a single-institution comparative experience. J Pediatr Surg. 2006;41(5): 966-71.

15. Koivusalo AI, Pakarinen MP, Rintala RJ, Saarinen-Pihkala UM. Surgical treatment of neuroblastoma: twenty-three years of experience at a single institution. Surg Today. 2013 .doi:10.1007/ s00595-013-0576-7.

16. Wang TI, Wu PK, Chen CF, Chen WM, Yen CC, Hung GY, et al. The prognosis of patients with primary osteosarcoma who have undergone unplanned therapy. Jpn J Clin Oncol 2011;41(11): 1244-50.

17. Hendershot E, Chang A, Colapinto K, Gerstle JT, Malkin D, Sung L. Postoperative fevers in pediatric solid tumor patients: how should they be managed? J Pediatr Hematol Oncol 2009;31(7): 485-8.

18. Ehrlich PF, Ritchey ML, Hamilton TE, Haase GM, Ou S, Breslow N, et al. Quality assessment for Wilms' tumor: a report from the National Wilms' Tumor Study-5. J Pediatr Surg 2005;40(1): 208-12 (discussion 12-3).

19. Wolff JE, Driever PH, Wolff B, Kramm CM, Kortmann RD, Pietsch T, et al. Pediatric neuro-oncology in small centersquality control of network support: the HIT-GBM experience. Anticancer Res. 2011;31(2):661-4. 